\title{
263.
}

\section{DEMONSTRATION OF A THEOREM IN FINITE DIFFERENCES.}

[From the Philosophical Transactions of the Royal Society of London, vol. CL. (for the year 1860), pp. 321-323: printed as a note to Sir J. W. F. Herschel's Memoir "On the Formulæ investigated by Dr BRINKLeEY for the general Term in the Development of Lagrange's Expression for the Summation of Series and for Successive Integrations, pp. 319-321.]

The formula (B) [of Sir J. W. F. Herscb sl's Memoir], substituting therein for $A_{x}$ the value $\frac{1}{[x]} \nabla^{-n} 0^{x}$, becomes

$$
\nabla^{-n} 0^{x}=-\frac{1}{[n-1]}\left\{\left[\begin{array}{l}
{[x+n]} \\
{[[x-1]}
\end{array} \frac{1}{[n+1]} 2.3 \ldots n \nabla-\frac{[x+n]}{[x-2]} \frac{1}{[n+2]} 3.4 \ldots \overline{n+1} \nabla^{2}-\& c .\right\} 0^{x}\right.
$$

or, as this may be written,

$$
\nabla^{-n} 0^{x}=\frac{[x+n]}{[n-1]}\left\{\quad\left\{\quad-\frac{1}{[1][x-1](n+1)} \nabla+\frac{1}{[2][x-2](n+2)} \nabla^{2}-\text { \&c. }\right\} 0^{x}\right.
$$

or, inserting a first term which vanishes except in the case $x=0$, and which is required in order that the formula may hold good for this particular value,

$$
\nabla^{-n} 0^{x}=\frac{[x+n]}{[n-1]}\left\{\frac{1}{[0][x] n} \nabla^{0}-\frac{1}{[1][x-1](n+1)} \nabla+\frac{1}{[2][x-2](n+2)} \nabla^{2}-\& c .\right\} 0^{x}
$$

where the series on the right-hand side need only be continued up to the term containing $\nabla^{x} 0^{x}$, since the subsequent terms vanish. [In these formulæ and throughout the present paper $[x]$ is written to denote the factorial $[x]^{x}$ or $\Pi(x)$, and so in other cases.]

Now $\nabla^{-n} 0^{x}$, or $\left(\frac{\Delta}{\log (1+\Delta)}\right)^{-n} 0^{x}$, is equal to $[x] \times$ coef. $t^{x}$ in $\left(\frac{e^{t}-1}{t}\right)^{-n}$, and so $\nabla^{q} 0^{x}$, or $\left(\frac{\Delta}{\log (1+\Delta)}\right)^{q} 0^{x}$, is equal to $[x] \times$ coef. $t^{x}$ in $\left(\frac{e^{t}-1}{t}\right)^{q}$. 
Hence, putting $R=\frac{e^{t}-1}{t}$, the last-mentioned formula will be true if, as regards the term which contains $t^{x}$, we have

$$
R^{-n}=\frac{[x+n]}{[n-1]}\left\{\frac{1}{[0][x] n} R^{0}-\frac{1}{[1][x-1](n+1)} R^{1}+\frac{1}{[2][x-2](n+2)} R^{2}-\& c .\right\},
$$

the series on the right-hand side being continued up to the term in $R^{x}$. This formula is, in fact, true if $R$, instead of being restricted to denote $\frac{e^{t}-1}{t}$, denotes any function whatever of the form $1+b t+c t^{2}+\& c$, , and it is true not only for the term in $t^{x}$, but for all the powers of $t$ not higher than $t^{x}$. And, moreover, $R^{-n}$ may denote any positive or negative integral or fractional power of $R$. In fact, the formula (assuming for a moment the truth of it) shows that the expansion of any power whatever of a series of the form in question, can be abtained by means of the expansions of the successive positive integer powers of the same series: the existence of such a formula (at least for negative powers) was indicated by Eisenstein, Crelle, t. xxxix. p. 181 (1850), and the formula itself, in a slightly different form, was obtained in a very simple manner by Professor Sylvester in his paper, "Development of an idea of Eisenstein," Quart. Math. Journ. t. I. p. 199 (1855); the demonstration was in fact as follows, viz. writing

$$
R^{n}=(1+\overline{R-1})^{n}=1+\frac{n}{1}(R-1)+\frac{n \cdot n-1}{1.2}(R-1)^{2}+\& c .:
$$

if we attend only to the terms involving powers of $t$ not higher than $t^{x}$, the series on the right-hand side needs only to be continued up to the term involving $(R-1)^{x}$, and the right side being thus converted into a rational and integral function of $R$, it may be developed in a series of powers of $R$ (the highest power being of course $R^{x}$ ), and the coefficients of the several powers are finite series which admit of summation; this gives the required formula. But there is an easier method; the process shows that the series on the right-hand side, continued as above up to the term involving $t^{x}$, is, as regards $n$, a rational and integral function of the degree $x$; and by Lagrange's interpolation formula, any rational and integral function of $n$ of the degree $x$, can be at once expressed in terms of the values corresponding to $x+1$ particular values of $n$. The investigation will be as follows:-Let $R$ denote a series of the form $1+b t+c t^{2}+\& c$., and let $R^{n}$ denote the development of the $n$th power of $R$, continued up to the term containing $t^{x}$, the terms involving higher powers of $t$ being rejected: $R^{0}, R^{1}, R^{2}$, \&c., and generally $R^{s}$, will in like manner denote the developments of these powers up to the term involving $t^{x}$, or what is the same thing, they will be the values of $R^{n}$, corresponding to $n=0,1,2, \ldots s$. By what precedes $R^{n}$ is a rational and integral function of $n$ of the degree $x$, and it can therefore be expressed in terms of the values $R^{0}, R^{1}, R^{2}, \ldots R^{x}$, which correspond to $n=0,1,2, \ldots x$. Let $s$ have any one of the last-mentioned values, then the expression

$$
\frac{n \cdot n-1 . n-2 \ldots n-x}{n-s} \frac{1}{s . s-1 \ldots 2 \cdot 1 \cdot-1 .-2 \ldots-(x-s)},
$$

which, as regards $n$, is a rational and integral function of the degree $x$ (the factor 
$n-s$, which occurs in the numerator and in the denominator being of course omitted), vanishes for each of the values $n=0,1,2, \ldots x$, except only for the value $n=s$, in which case it becomes equal to unity. The required formula is thus seen to be

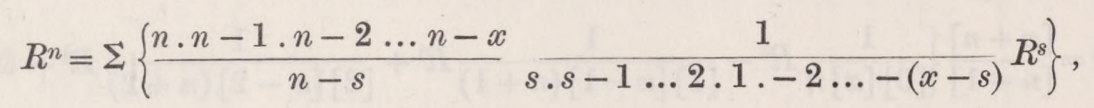

where the summation extends to the several values $s=0,1,2, \ldots x$; or, what is the same thing, it is

$$
R^{n}=\Sigma\left\{\frac{n \cdot n-1 \cdot n-2 \ldots n-x}{n-s} \frac{(-)^{x-s}}{1.2 \ldots s \cdot 1 \cdot 2 \ldots(x-s)} R^{s}\right\}
$$

or changing the sign of $n$, it is

$$
R^{-n}=\Sigma\left\{\frac{n \cdot n+1 \cdot n+2 \ldots n+x}{n+s} \frac{(-)^{s}}{1.2 \ldots s .1 .2 \ldots x-s} R^{s}\right\}
$$

or, as this may be written,

$$
R^{-n}=\frac{[x+n]}{[n-1]} \Sigma\left\{\frac{(-)^{s}}{[s][x-s](n+s)} R^{s}\right\}
$$

or substituting for $s$ the values $0,1,2, \ldots x$, the formula is

$$
R^{-n}=\frac{[x+n]}{[n-1]}\left\{\frac{1}{[0][x] n} R^{0}-\frac{1}{[1][x-1](n+1)} R^{1}+\frac{1}{[2][x-2](n+2)} R^{2}-. .\right\}
$$

continued up to the term involving $R^{x}$, which is the theorem in question. 